\title{
Three-point boundary value problems of fractional functional differential equations with delay
}

\author{
Yanan Li, Shurong Sun *, Dianwu Yang and Zhenlai Han
}

${ }^{\text {"Correspondence: }}$ sshrong@163.com School of Mathematical Sciences, University of Jinan, Jinan, Shandong 250022, P.R. China

\begin{abstract}
In this paper, we study three-point boundary value problems of the following fractional functional differential equations involving the Caputo fractional derivative:

$$
\begin{aligned}
& { }^{C} D^{\alpha} u(t)=f\left(t, u_{t}{ }^{C} D^{\beta} u(t)\right), \quad 0<t<1, \\
& u^{\prime}(0)=0, \quad u^{\prime}(1)=\lambda u^{\prime}(\eta),
\end{aligned}
$$

where ${ }^{C} D^{\alpha},{ }^{C} D^{\beta}$ denote Caputo fractional derivatives, $2<\alpha<3,0<\beta<1, \eta \in(0,1)$, $1<\lambda<\frac{1}{2 \eta}$. We use the Green function to reformulate boundary value problems into an abstract operator equation. By means of the Schauder fixed point theorem and the Banach contraction principle, some existence results of solutions are obtained, respectively. As an application, some examples are presented to illustrate the main results.
\end{abstract}

MSC: $34 \mathrm{~A} 08 ; 34 \mathrm{~K} 37$

Keywords: fractional functional differential equation; delay; three-point boundary value problems; fixed point theorem; existence of solutions

\section{Introduction}

Fractional calculus is a branch of mathematics, it is an emerging field in the area of the applied mathematics that deals with derivatives and integrals of arbitrary orders as well as with their applications. The origins can be traced back to the end of the seventeenth century. During the history of fractional calculus, it was reported that the pure mathematical formulations of the investigated problems started to be addressed with more applications in various fields. With the help of fractional calculus, we can describe natural phenomena and mathematical models more accurately. Therefore, fractional differential equations have received much attention and the theory and its application have been greatly developed; see [1-6].

Recently, there have been many papers focused on boundary value problems of fractional ordinary differential equations [7-15] and an initial value problem of fractional functional differential equations [16-28]. But the results dealing with the boundary value problems of fractional functional differential equations with delay are relatively scarce [29-35]. It is well known that in practical problems, the behavior of systems not only depends on the status just at the present, but also on the status in the past. Thus, in many 
cases, we must consider fractional functional differential equations with delay in order to solve practical problems. Consequently, our aim in this paper is to study the existence of solutions for boundary value problems of fractional functional differential equations.

In 2011, Rehman [12] studied the existence and uniqueness of solutions to nonlinear three-point boundary value problems for the following fractional differential equation:

$$
\begin{aligned}
& { }^{C} D_{0^{+}}^{\delta} u(t)=f\left(t, u(t),{ }^{C} D_{0^{+}}^{\sigma} u(t)\right), \quad t \in[0, T], \\
& u(0)=\alpha u(\eta), \quad u(T)=\beta u(\eta),
\end{aligned}
$$

where $1<\delta<2,0<\sigma<1, \alpha, \beta \in \mathbb{R}, \alpha \eta(1-\beta)+(1-\alpha)(t-\beta \eta) \neq 0$ and ${ }^{C} D_{0^{+}}^{\delta},{ }^{C} D_{0^{+}}^{\sigma}$ denote Caputo fractional derivatives. By the Banach contraction principle and the Schauder fixed point theorem, they obtained some new existence and uniqueness results.

For $0<r<1$, we denote by $C_{r}$ the Banach space of all continuous functions $\varphi:[-r, 0] \rightarrow$ $\mathbb{R}$ endowed with the sup-norm

$$
\|\varphi\|_{[-r, 0]}:=\sup \{|\varphi(s)|: s \in[-r, 0]\} .
$$

If $u:[-r, 1] \rightarrow \mathbb{R}$, then for any $t \in[0,1]$, we denote by $u_{t}$ the element of $C_{r}$ defined by

$$
u_{t}(\theta)=u(t+\theta), \quad \text { for } \theta \in[-r, 0] .
$$

Enlightened by literature [12], in this paper we study the following three-point boundary value problem for the fractional functional differential equation:

$$
{ }^{C} D^{\alpha} u(t)=f\left(t, u_{t},{ }^{C} D^{\beta} u(t)\right), \quad 0<t<1,
$$

where $2<\alpha<3,0<\beta<1$ and ${ }^{C} D^{\alpha},{ }^{C} D^{\beta}$ denote Caputo fractional derivatives, $f\left(t, u_{t}\right.$, $\left.{ }^{C} D^{\beta} u(t)\right)$ is a continuous function associated with the boundary conditions

$$
u^{\prime}(0)=0, \quad u^{\prime}(1)=\lambda u^{\prime}(\eta)
$$

and $u_{0}=\varphi$, where $\eta \in(0,1), 1<\lambda<\frac{1}{2 \eta}$ and $\varphi$ is an element of the space

$$
C_{r}^{+}(0):=\left\{\psi \in C_{r} \mid \psi(s) \geq 0, s \in[-r, 0], \psi(0)=0,{ }^{C} D^{\beta} \psi(s)=0\right\} .
$$

To the best of our knowledge, no one has studied the existence of positive solutions for problem (1.1)-(1.2). The aim of this paper is to fill the gap in the relevant literatures. In this paper, we firstly give the fractional Green function and some properties of the Green function. Consequently, boundary value problem (1.1) and (1.2) is reduced to an equivalent Fredholm integral equation. Then we extend the existence results for boundary value problems of an ordinary fractional differential equation of $\delta$-order $(1<\delta<2)$ in [12] to a fractional functional differential equation of $\alpha$-order $(2<\alpha<3)$. As an application, some examples are presented to illustrate the main results. 


\section{Preliminaries}

For the convenience of the reader, we give the following background material from fractional calculus theory to facilitate the analysis of boundary value problem (1.1) and (1.2). This material can be found in the recent literature; see $[1,2,36]$.

Definition 2.1 ([1]) The fractional integral of order $\alpha(\alpha>0)$ of a function $f:\left(t_{0},+\infty\right) \rightarrow$ $\mathbb{R}$ is given by

$$
I^{\alpha} f(t)=\frac{1}{\Gamma(\alpha)} \int_{t_{0}}^{t} \frac{f(s)}{(t-s)^{1-\alpha}} d s, \quad t>t_{0},
$$

where $\Gamma(\cdot)$ is the gamma function, provided that the right-hand side is point-wise defined on $\left(t_{0},+\infty\right)$.

Definition 2.2 ([1]) The Caputo fractional derivative of order $\alpha(n-1<\alpha<n)$ of a function $f:\left(t_{0},+\infty\right) \rightarrow \mathbb{R}$ is given by

$$
{ }^{C} D^{\alpha} f(t)=\frac{1}{\Gamma(n-\alpha)} \int_{t_{0}}^{t} \frac{f^{(n)}(s)}{(t-s)^{\alpha+1-n}} d s, \quad t>t_{0},
$$

where $\Gamma(\cdot)$ is the gamma function, provided that the right-hand side is point-wise defined on $\left(t_{0},+\infty\right)$.

Obviously, the Caputo derivative for every constant function is equal to zero.

From the definition of the Caputo derivative, we can acquire the following statement.

Lemma $2.1([2])$ Let $f(t) \in L^{1}\left[t_{0}, \infty\right)$. Then

$$
{ }^{C} D^{\alpha}\left(I^{\alpha} f(t)\right)=f(t), \quad t>t_{0} \text { and } 0<\alpha<1 .
$$

Lemma $2.2([2])$ Let $\alpha>0$. Then

$$
I^{\alpha C} D^{\alpha} f(t)=f(t)-c_{1}-c_{2} t-\cdots-c_{n} t^{n-1}
$$

for some $c_{i} \in \mathbb{R}, i=1,2, \ldots, n$, where $n=[\alpha]+1$ and $[\alpha]$ denotes the integer part of $\alpha$.

Next, we introduce the Green function of fractional functional differential equations boundary value problems.

Lemma 2.3 Let $2<\alpha<3,0<\eta<1,1<\lambda<\frac{1}{2 \eta}$ and $h:[0,1] \rightarrow \mathbb{R}$ be continuous. Then the boundary value problem

$$
\begin{aligned}
& { }^{C} D^{\alpha} u(t)=h(t), \quad 0<t<1, \\
& u(0)=u^{\prime}(0)=0, \quad u^{\prime}(1)=\lambda u^{\prime}(\eta),
\end{aligned}
$$

has a unique solution

$$
u(t)=\int_{0}^{1} G(t, s) h(s) d s
$$


where

$$
\begin{aligned}
& G(t, s) \\
& \quad=\frac{1}{\Gamma(\alpha)} \begin{cases}(t-s)^{\alpha-1}+\frac{(\alpha-1) t^{2}}{2-2 \lambda \eta}\left((1-s)^{\alpha-2}-\lambda(\eta-s)^{\alpha-2}\right), & 0 \leq s \leq t \leq 1, s \leq \eta \\
(t-s)^{\alpha-1}+\frac{(\alpha-1) t^{2}}{2-2 \lambda \eta}(1-s)^{\alpha-2}, & 0 \leq s \leq t \leq 1, \eta \leq s, \\
\frac{(\alpha-1) t^{2}}{2-2 \lambda \eta}\left((1-s)^{\alpha-2}-\lambda(\eta-s)^{\alpha-2}\right), & 0 \leq t \leq s \leq 1, s \leq \eta, \\
\frac{(\alpha-1) t^{2}}{2-2 \lambda \eta}(1-s)^{\alpha-2}, & 0 \leq t \leq s \leq 1, \eta \leq s .\end{cases}
\end{aligned}
$$

Proof From equation (2.1), we know

$$
I^{\alpha C} D^{\alpha} u(t)=I^{\alpha} h(t)
$$

From Lemma 2.2, we have

$$
\begin{aligned}
& u(t)-c_{1}-c_{2} t-c_{3} t^{2}=\frac{1}{\Gamma(\alpha)} \int_{0}^{t}(t-s)^{\alpha-1} h(s) d s, \\
& u(t)=\frac{1}{\Gamma(\alpha)} \int_{0}^{t}(t-s)^{\alpha-1} h(s) d s+c_{1}+c_{2} t+c_{3} t^{2} .
\end{aligned}
$$

According to (2.1), we know that

$$
\begin{aligned}
& c_{1}=c_{2}=0, \\
& u^{\prime}(t)=\frac{\alpha-1}{\Gamma(\alpha)} \int_{0}^{t}(t-s)^{\alpha-2} h(s) d s+2 c_{3} t .
\end{aligned}
$$

By $u^{\prime}(1)=\lambda u^{\prime}(\eta)$, we have

$$
c_{3}=\frac{\alpha-1}{(2-2 \lambda \eta) \Gamma(\alpha)}\left(\int_{0}^{1}(1-s)^{\alpha-2} h(s) d s-\lambda \int_{0}^{\eta}(\eta-s)^{\alpha-2} h(s) d s\right) .
$$

Therefore,

$$
\begin{aligned}
u(t)= & \frac{1}{\Gamma(\alpha)} \int_{0}^{t}(t-s)^{\alpha-1} h(s) d s \\
& +\frac{(\alpha-1) t^{2}}{(2-2 \lambda \eta) \Gamma(\alpha)}\left(\int_{0}^{1}(1-s)^{\alpha-2} h(s) d s-\lambda \int_{0}^{\eta}(\eta-s)^{\alpha-2} h(s) d s\right), \\
u^{\prime}(t)= & \frac{\alpha-1}{\Gamma(\alpha)} \int_{0}^{t}(t-s)^{\alpha-2} h(s) d s \\
& +\frac{2 t(\alpha-1)}{(2-2 \lambda \eta) \Gamma(\alpha)}\left(\int_{0}^{1}(1-s)^{\alpha-2} h(s) d s-\lambda \int_{0}^{\eta}(\eta-s)^{\alpha-2} h(s) d s\right) .
\end{aligned}
$$

Now, for $t \leq \eta$, we have

$$
\begin{aligned}
u(t)= & \frac{1}{\Gamma(\alpha)} \int_{0}^{t}(t-s)^{\alpha-1} h(s) d s \\
& +\frac{(\alpha-1) t^{2}}{(2-2 \lambda \eta) \Gamma(\alpha)}\left(\left(\int_{0}^{t}+\int_{t}^{\eta}+\int_{\eta}^{1}\right)(1-s)^{\alpha-2} h(s) d s\right.
\end{aligned}
$$




$$
\begin{aligned}
& \left.-\lambda\left(\int_{0}^{t}+\int_{t}^{\eta}\right)(\eta-s)^{\alpha-2} h(s) d s\right) \\
= & \frac{1}{\Gamma(\alpha)} \int_{0}^{t}\left((t-s)^{\alpha-1}+\frac{(\alpha-1) t^{2}}{2-2 \lambda \eta}\left((1-s)^{\alpha-2}-\lambda(\eta-s)^{\alpha-2}\right)\right) h(s) d s \\
& +\frac{1}{\Gamma(\alpha)} \int_{t}^{\eta} \frac{(\alpha-1) t^{2}}{2-2 \lambda \eta}\left((1-s)^{\alpha-2}-\lambda(\eta-s)^{\alpha-2}\right) h(s) d s \\
& +\frac{1}{\Gamma(\alpha)} \int_{\eta}^{1} \frac{(\alpha-1) t^{2}}{2-2 \lambda \eta}(1-s)^{\alpha-2} h(s) d s .
\end{aligned}
$$

For $t \geq \eta$, we have

$$
\begin{aligned}
u(t)= & \frac{1}{\Gamma(\alpha)}\left(\int_{0}^{\eta}+\int_{\eta}^{t}\right)(t-s)^{\alpha-1} h(s) d s \\
& +\frac{(\alpha-1) t^{2}}{(2-2 \lambda \eta) \Gamma(\alpha)}\left(\left(\int_{0}^{\eta}+\int_{\eta}^{t}+\int_{t}^{1}\right)(1-s)^{\alpha-2} h(s) d s-\lambda \int_{0}^{\eta}(\eta-s)^{\alpha-2} h(s) d s\right) \\
= & \frac{1}{\Gamma(\alpha)} \int_{0}^{\eta}\left((t-s)^{\alpha-1}+\frac{(\alpha-1) t^{2}}{2-2 \lambda \eta}\left((1-s)^{\alpha-2}-\lambda(\eta-s)^{\alpha-2}\right)\right) h(s) d s \\
& +\frac{1}{\Gamma(\alpha)} \int_{\eta}^{t}\left((t-s)^{\alpha-1}+\frac{(\alpha-1) t^{2}}{2-2 \lambda \eta}(1-s)^{\alpha-2}\right) h(s) d s \\
& +\frac{1}{\Gamma(\alpha)} \int_{t}^{1} \frac{(\alpha-1) t^{2}}{2-2 \lambda \eta}(1-s)^{\alpha-2} h(s) d s .
\end{aligned}
$$

Hence, we can conclude (2.2) holds, where

$$
G(t, s)=\frac{1}{\Gamma(\alpha)} \begin{cases}(t-s)^{\alpha-1}+\frac{(\alpha-1) t^{2}}{2-2 \lambda \eta}\left((1-s)^{\alpha-2}-\lambda(\eta-s)^{\alpha-2}\right), & 0 \leq s \leq t \leq 1, s \leq \eta, \\ (t-s)^{\alpha-1}+\frac{(\alpha-1) t^{2}}{2-2 \lambda \eta}(1-s)^{\alpha-2}, & 0 \leq s \leq t \leq 1, \eta \leq s, \\ \frac{(\alpha-1) t^{2}}{2-2 \lambda \eta}\left((1-s)^{\alpha-2}-\lambda(\eta-s)^{\alpha-2}\right), & 0 \leq t \leq s \leq 1, s \leq \eta, \\ \frac{(\alpha-1) t^{2}}{2-2 \lambda \eta}(1-s)^{\alpha-2}, & 0 \leq t \leq s \leq 1, \eta \leq s .\end{cases}
$$

The proof is completed.

Lemma 2.4 ([36] Schauder fixed point theorem) Let $(D, d)$ be a complete metric space, $U$ be a closed convex subset of $D$, and $T: D \rightarrow D$ be the map such that the set $T u: u \in U$ is relatively compact in $D$. Then the operator $T$ has at least one fixed point $u^{\prime \prime} \in U$ :

$$
T u^{*}=u^{*}
$$

\section{Main results}

In this section, we discuss the existence and uniqueness of solutions for boundary value problem (1.1) and (1.2) by the Schauder fixed point theorem and the Banach contraction principle.

For convenience, we define the Banach space $X=\left\{u \mid u \in C[-r, 1],{ }^{C} D^{\beta} u \in C[-r, 1], 0<\right.$ $\beta<1\}$. Also, if $I$ is an interval of the real line $\mathbb{R}$, by $C(I)$ and $C^{1}(I)$ we denote the set of continuous and continuously differentiable functions on $I$, respectively. Moreover, for $u \in$ 
$C(I)$, we define

$$
\|u\|_{I}=\max _{t \in I}|u(t)|+\max _{t \in I}\left|{ }^{C} D^{\beta} u(t)\right| .
$$

For $u_{0}=\varphi$, in view of the definitions of $u_{t}$ and $\varphi$, we have

$$
u_{0}=u(\theta)=\varphi(\theta), \quad \text { for } \theta \in[-r, 0]
$$

Thus, we have

$$
u(t)=\varphi(t), \quad \text { for } t \in[-r, 0] .
$$

Since $f:[0,1] \times C_{r} \times \mathbb{R} \rightarrow \mathbb{R}$ is a continuous function, set $f\left(t, u_{t},{ }^{C} D^{\beta} u(t)\right):=h(t)$ in Lemma 2.3. We have by Lemma 2.3 that a function $u$ is a solution of boundary value problem (1.1) and (1.2) if and only if it satisfies

$$
u(t)= \begin{cases}\int_{0}^{1} G(t, s) f\left(s, u_{s},{ }^{C} D^{\beta} u(s)\right) d s, & t \in(0,1), \\ \varphi(t), & t \in[-r, 0] .\end{cases}
$$

We define an operator $T: X \rightarrow X$ as follows:

$$
T u(t)=u(t)= \begin{cases}\int_{0}^{1} G(t, s) f\left(s, u_{s},{ }^{C} D^{\beta} u(s)\right) d s, & t \in(0,1), \\ \varphi(t), & t \in[-r, 0],\end{cases}
$$

and

$$
\begin{aligned}
& l=\max _{0 \leq t \leq 1}\left(\int_{0}^{1}|G(t, s) g(s)| d s\right), \\
& l^{*}=\max _{0 \leq t \leq 1}\left(\int_{0}^{1}\left|\frac{\partial}{\partial t} G(t, s) g(s)\right| d s\right), \\
& Q=\frac{1}{\Gamma(2-\beta)}+\frac{1+\lambda \eta^{\alpha-1}}{\Gamma(2-\beta)(1-\lambda \eta)}+\frac{1}{\alpha}+\frac{\lambda \eta^{\alpha-1}+1}{2-2 \lambda \eta} .
\end{aligned}
$$

Theorem 3.1 Assume the following:

$\left(\mathrm{H}_{1}\right)$ There exists a nonnegative function $g \in L[0,1]$ such that

$$
|f(t, v, w)| \leq g(t)+a|v|^{k_{1}}+b|w|^{k_{2}}
$$

for each $v \in C_{r}, w \in \mathbb{R}$, where $a, b \in \mathbb{R}$ are nonnegative constants and $0<k_{1}, k_{2}<1$; or

$\left(\mathrm{H}_{2}\right)$ There exists a nonnegative function $g \in L[0,1]$ such that

$$
|f(t, v, w)| \leq g(t)+a|v|^{k_{1}}+b|w|^{k_{2}}
$$

for each $v \in C_{r}, w \in \mathbb{R}$, where $a, b \in \mathbb{R}$ are nonnegative constants and $k_{1}, k_{2}>1$.

Then boundary value problem (1.1) and (1.2) has a solution. 
Proof Suppose $\left(\mathrm{H}_{1}\right)$ holds. Choose

$$
\omega \geq \max \left\{3\left(l+\frac{l^{*}}{\Gamma(2-\beta)}\right),(3 a Q)^{\frac{1}{1-k_{1}}},(3 b Q)^{\frac{1}{1-k_{2}}}\right\}
$$

and define the cone $U=\{u \in X \mid\|u\| \leq \omega, \omega>0\}$.

For any $u \in U$, we have

$$
\begin{aligned}
|T u(t)|= & \left|\int_{0}^{1} G(t, s) f\left(s, u_{s},{ }^{C} D^{\beta} u(s)\right) d s\right| \\
\leq & \int_{0}^{1}|G(t, s) g(s)| d s+\left(a|\omega|^{k_{1}}+b|\omega|^{k_{2}}\right)\left(\int_{0}^{t} \frac{(t-s)^{\alpha-1}}{\Gamma(\alpha)} d s\right. \\
& \left.+\frac{(\alpha-1) t^{2}}{2-2 \lambda \eta} \int_{0}^{1} \frac{(1-s)^{\alpha-2}}{\Gamma(\alpha)} d s+\frac{(\alpha-1) \lambda t^{2}}{2-2 \lambda \eta} \int_{0}^{\eta} \frac{(\eta-s)^{\alpha-2}}{\Gamma(\alpha)} d s\right) \\
\leq & l+\left(a|\omega|^{k_{1}}+b|\omega|^{k_{2}}\right)\left(\frac{t^{\alpha}}{\alpha \Gamma(\alpha)}+\frac{t^{2}}{(2-2 \lambda \eta) \Gamma(\alpha)}+\frac{\lambda t^{2} \eta^{\alpha-1}}{(2-2 \lambda \eta) \Gamma(\alpha)}\right) \\
\leq & l+\frac{a|\omega|^{k_{1}}+b|\omega|^{k_{2}}}{\Gamma(\alpha)}\left(\frac{1}{\alpha}+\frac{\lambda \eta^{\alpha-1}+1}{2-2 \lambda \eta}\right) .
\end{aligned}
$$

Also,

$$
\begin{aligned}
\left|T u^{\prime}(t)\right| \leq & \int_{0}^{1}\left|\frac{\partial}{\partial t} G(t, s)\right| f\left(s, u_{s},{ }^{C} D^{\beta} u(s)\right) \mid d s \\
\leq & \int_{0}^{1}\left|\frac{\partial}{\partial t} G(t, s) g(s)\right| d s+\left(a|\omega|^{k_{1}}+b|\omega|^{k_{2}}\right)\left(\int_{0}^{t} \frac{(t-s)^{\alpha-2}}{\Gamma(\alpha-1)} d s\right. \\
& \left.+\frac{2(\alpha-1) t}{2-2 \lambda \eta} \int_{0}^{1} \frac{(1-s)^{\alpha-2}}{\Gamma(\alpha)} d s+\frac{2(\alpha-1) \lambda t}{2-2 \lambda \eta} \int_{0}^{\eta} \frac{(\eta-s)^{\alpha-2}}{\Gamma(\alpha)} d s\right) \\
\leq & l^{*}+\left(a|\omega|^{k_{1}}+b|\omega|^{k_{2}}\right)\left(\frac{t^{\alpha-1}}{\Gamma(\alpha)}+\frac{t}{(1-\lambda \eta) \Gamma(\alpha)}+\frac{\lambda t \eta^{\alpha-1}}{(1-\lambda \eta) \Gamma(\alpha)}\right) \\
\leq & l^{*}+\frac{a|\omega|^{k_{1}}+b|\omega|^{k_{2}}}{\Gamma(\alpha)}\left(1+\frac{1+\lambda \eta^{\alpha-1}}{1-\lambda \eta}\right) .
\end{aligned}
$$

Hence,

$$
\begin{aligned}
\mid{ }^{C} & D^{\beta} T u(t) \mid \\
& \leq \frac{1}{\Gamma(1-\beta)} \int_{0}^{t}(t-s)^{-\beta}\left|T u^{\prime}(s)\right| d s \\
& \leq l^{*} \frac{t^{1-\beta}}{(1-\beta) \Gamma(1-\beta)}+\frac{\left(a|\omega|^{k_{1}}+b|\omega|^{k_{2}}\right) t^{1-\beta}}{\Gamma(2-\beta)}\left(\frac{t^{\alpha-1}}{\Gamma(\alpha)}+\frac{t}{(1-\lambda \eta) \Gamma(\alpha)}+\frac{\lambda t \eta^{\alpha-1}}{(1-\lambda \eta) \Gamma(\alpha)}\right) \\
& \leq \frac{l^{*}}{\Gamma(2-\beta)}+\frac{a|\omega|^{k_{1}}+b|\omega|^{k_{2}}}{\Gamma(2-\beta) \Gamma(\alpha)}\left(1+\frac{1+\lambda \eta^{\alpha-1}}{1-\lambda \eta}\right) .
\end{aligned}
$$

In view of (3.1) and (3.3), we obtain

$$
\begin{aligned}
\|T u(t)\| \leq & l+\frac{l^{*}}{\Gamma(2-\beta)} \\
& +\frac{a|\omega|^{k_{1}}+b|\omega|^{k_{2}}}{\Gamma(\alpha)}\left(\frac{1}{\Gamma(2-\beta)}+\frac{1+\lambda \eta^{\alpha-1}}{\Gamma(2-\beta)(1-\lambda \eta)}+\frac{1}{\alpha}+\frac{\lambda \eta^{\alpha-1}+1}{2-2 \lambda \eta}\right)
\end{aligned}
$$




$$
\begin{aligned}
& \leq \frac{\omega}{3}+\left(a|\omega|^{k_{1}}+b|\omega|^{k_{2}}\right) Q \\
& \leq \frac{\omega}{3}+\frac{\omega}{3}+\frac{\omega}{3} \\
& \leq \omega
\end{aligned}
$$

which implies that $T: U \rightarrow U$. The continuity of the operator $T$ follows from the continuity of $f$ and $G$.

Now, if $\left(\mathrm{H}_{2}\right)$ holds, we choose

$$
0<\omega \leq \min \left\{3\left(l+\frac{l^{*}}{\Gamma(2-\beta)}\right),\left(\frac{1}{3 a Q}\right)^{\frac{1}{1-k_{1}}},\left(\frac{1}{3 b Q}\right)^{\frac{1}{1-k_{2}}}\right\}
$$

and by the same process as above, we obtain

$$
\begin{aligned}
\|T u(t)\| \leq & l+\frac{l^{*}}{\Gamma(2-\beta)} \\
& +\frac{a|\omega|^{k_{1}}+b|\omega|^{k_{2}}}{\Gamma(\alpha)}\left(\frac{1}{\Gamma(2-\beta)}+\frac{1+\lambda \eta^{\alpha-1}}{\Gamma(2-\beta)(1-\lambda \eta)}+\frac{1}{\alpha}+\frac{\lambda \eta^{\alpha-1}+1}{2-2 \lambda \eta}\right) \\
\leq & \frac{\omega}{3}+\left(a|\omega|^{k_{1}}+b|\omega|^{k_{2}}\right) Q \\
\leq & \frac{\omega}{3}+\frac{\omega}{3}+\frac{\omega}{3} \\
\leq & \omega,
\end{aligned}
$$

which implies that $T: U \rightarrow U$.

Now, we show that $T$ is a completely continuous operator.

Let $L=\max _{0 \leq t \leq 1}\left|f\left(t, u_{t},{ }^{C} D^{\beta} u(t)\right)\right|+1$. Then for $u \in U$ and $t_{1}, t_{2} \in[-r, 1]$ with $t_{1}<t_{2}$, in view of Lemma 2.3, if $0 \leq t_{1}<t_{2} \leq 1$, then

$$
\begin{aligned}
\left|T u\left(t_{2}\right)-T u\left(t_{1}\right)\right| & \left|\int_{0}^{1} G\left(t_{2}, s\right) f\left(s, u_{s},{ }^{C} D^{\beta} u(s)\right) d s-\int_{0}^{1} G\left(t_{1}, s\right) f\left(s, u_{s},{ }^{C} D^{\beta} u(s)\right) d s\right| \\
\leq & \int_{0}^{t_{1}}\left|G\left(t_{2}, s\right)-G\left(t_{1}, s\right)\right| L d s+\int_{t_{2}}^{1}\left|G\left(t_{2}, s\right)-G\left(t_{1}, s\right)\right| L d s \\
& +\int_{t_{1}}^{t_{2}}\left|G\left(t_{2}, s\right)-G\left(t_{1}, s\right)\right| L d s \\
< & \frac{L}{\Gamma(\alpha)} \mid \int_{0}^{t_{1}}\left(\left(t_{2}-s\right)^{\alpha-1}-\left(t_{1}-s\right)^{\alpha-1}\right. \\
& \left.+\frac{(\alpha-1)(1-s)^{\alpha-2}\left(t_{2}^{2}-t_{1}^{2}\right)}{2-2 \lambda \eta}-\frac{\lambda(\eta-s)^{\alpha-2}(\alpha-1)\left(t_{2}^{2}-t_{1}^{2}\right)}{2-2 \lambda \eta}\right) d s \\
& +\int_{t_{2}}^{1} \frac{(\alpha-1)\left(t_{2}^{2}-t_{1}^{2}\right)}{2-2 \lambda \eta}\left((1-s)^{\alpha-2}-\lambda(\eta-s)^{\alpha-2}\right) d s \\
& +\int_{t_{1}}^{t_{2}} \frac{(\alpha-1)\left(t_{2}^{2}-t_{1}^{2}\right)}{2-2 \lambda \eta}\left((1-s)^{\alpha-2}-\lambda(\eta-s)^{\alpha-2}\right)-\left(t_{2}-s\right)^{\alpha-1} d s \mid \\
\leq & \frac{L}{\Gamma(\alpha)} \mid \int_{0}^{1} \frac{(\alpha-1)\left(t_{2}^{2}-t_{1}^{2}\right)(1-s)^{\alpha-2}}{2-2 \lambda \eta} d s-\int_{0}^{\eta} \frac{(\alpha-1)\left(t_{2}^{2}-t_{1}^{2}\right) \lambda(\eta-s)^{\alpha-2}}{2-2 \lambda \eta} d s
\end{aligned}
$$




$$
\begin{aligned}
& +\int_{0}^{t_{1}}\left(t_{1}-s\right)^{\alpha-1} d s-\int_{0}^{t_{2}}\left(t_{2}-s\right)^{\alpha-1} d s \mid \\
\leq & \frac{L}{\Gamma(\alpha)}\left|\frac{t_{2}^{\alpha}-t_{1}^{\alpha}}{\alpha}+\frac{\left(t_{2}^{2}-t_{1}^{2}\right)\left(1-\lambda \eta^{\alpha-1}\right)}{2-2 \lambda \eta}\right| .
\end{aligned}
$$

If $-r \leq t_{1}<t_{2} \leq 0$, then

$$
\left|T u\left(t_{2}\right)-T u\left(t_{1}\right)\right|=\left|\varphi\left(t_{2}\right)-\varphi\left(t_{1}\right)\right| .
$$

If $-r \leq t_{1}<0<t_{2} \leq 1$, then

$$
\begin{aligned}
\left|T u\left(t_{2}\right)-T u\left(t_{1}\right)\right| & \leq\left|T u\left(t_{2}\right)-T u(0)\right|+\left|T u(0)-T u\left(t_{1}\right)\right| \\
& \leq \int_{0}^{1}\left|G\left(t_{2}, s\right)-G(0, s)\right| f\left(s, u_{s},{ }^{C} D^{\beta} u(s)\right)|d s+| \varphi(0)-\varphi\left(t_{1}\right) \mid \\
& \leq \frac{L}{\Gamma(\alpha)}\left|\frac{t_{2}^{\alpha}}{\alpha}+\frac{t_{2}^{2}\left(1-\lambda \eta^{\alpha-1}\right)}{2-2 \lambda \eta}\right|+\varphi\left(t_{1}\right) .
\end{aligned}
$$

Hence, if $0 \leq t_{1}<t_{2} \leq 1$, we have

$$
\begin{aligned}
\left|{ }^{C} D^{\beta} T u\left(t_{2}\right)-{ }^{C} D^{\beta} T u\left(t_{1}\right)\right| \\
=\frac{1}{\Gamma(1-\beta)}\left|\int_{0}^{t_{2}}\left(t_{2}-s\right)^{-\beta} T u^{\prime}(s) d s-\int_{0}^{t_{1}}\left(t_{1}-s\right)^{-\beta} T u^{\prime}(s) d s\right| \\
\leq \frac{1}{\Gamma(1-\beta)}\left|\int_{0}^{t_{2}}\left(t_{2}-s\right)^{-\beta} T u^{\prime}(s) d s-\int_{0}^{t_{1}}\left(t_{2}-s\right)^{-\beta} T u^{\prime}(s) d s\right| \\
\quad+\frac{1}{\Gamma(1-\beta)}\left|\int_{0}^{t_{1}}\left(t_{2}-s\right)^{-\beta} T u^{\prime}(s) d s-\int_{0}^{t_{1}}\left(t_{1}-s\right)^{-\beta} T u^{\prime}(s) d s\right| \\
\leq \frac{1}{\Gamma(1-\beta)}\left|\int_{t_{1}}^{t_{2}}\left(t_{2}-s\right)^{-\beta}\right| T u^{\prime}(s)\left|d s+\int_{0}^{t_{1}}\left(\left(t_{2}-s\right)^{-\beta}-\left(t_{1}-s\right)^{-\beta}\right)\right| T u^{\prime}(s)|d s| \\
\leq \frac{1}{\Gamma(1-\beta)} \mid \int_{t_{1}}^{t_{2}}\left(t_{2}-s\right)^{-\beta}\left(\int_{0}^{1}\left|\frac{\partial}{\partial s} G(s, z)\right| f\left(z, u_{z},{ }^{C} D^{\beta} u(z)\right) \mid d z\right) d s \\
\quad+\int_{0}^{t_{1}}\left(\left(t_{2}-s\right)^{-\beta}-\left(t_{1}-s\right)^{-\beta}\right)\left(\int_{0}^{1}\left|\frac{\partial}{\partial s} G(s, z)\right|\left|f\left(z, u_{z},{ }^{C} D^{\beta} u(z)\right)\right| d z\right) d s \mid \\
\leq \frac{L\left(2-\lambda \eta+\lambda \eta^{\alpha-1}\right)}{\Gamma(1-\beta)(1-\lambda \eta) \Gamma(\alpha)}\left|\int_{t_{1}}^{t_{2}}\left(t_{2}-s\right)^{-\beta} d s+\int_{0}^{t_{1}}\left(\left(t_{2}-s\right)^{-\beta}-\left(t_{1}-s\right)^{-\beta}\right) d s\right| \\
\leq \frac{L\left(2-\lambda \eta+\lambda \eta^{\alpha-1}\right)}{(1-\lambda \eta) \Gamma(2-\beta) \Gamma \alpha)}\left|t_{2}^{1-\beta}-t_{1}^{1-\beta}\right| .
\end{aligned}
$$

If $-r \leq t_{1}<t_{2} \leq 0$, in view of the definition of $\varphi$, we have

$$
\left|{ }^{C} D^{\beta} T u\left(t_{2}\right)-{ }^{C} D^{\beta} T u\left(t_{1}\right)\right|=\left|{ }^{C} D^{\beta} \varphi\left(t_{2}\right)-{ }^{C} D^{\beta} \varphi\left(t_{1}\right)\right|=0 .
$$

If $-r \leq t_{1}<0<t_{2} \leq 1$, then

$$
\begin{aligned}
& \left|{ }^{C} D^{\beta} T u\left(t_{2}\right)-{ }^{C} D^{\beta} T u\left(t_{1}\right)\right| \\
& \quad=\frac{1}{\Gamma(1-\beta)}\left|\int_{0}^{t_{2}}\left(t_{2}-s\right)^{-\beta} T u^{\prime}(s) d s\right|-0
\end{aligned}
$$




$$
\begin{aligned}
& \leq \frac{1}{\Gamma(1-\beta)}\left|\int_{0}^{t_{2}}\left(t_{2}-s\right)^{-\beta}\left(\int_{0}^{1}\left|\frac{\partial}{\partial s} G(s, z)\right| f\left(z, u_{z},{ }^{C} D^{\beta} u(z)\right) \mid d z\right) d s\right| \\
& \leq \frac{L t_{2}^{1-\beta}}{\Gamma(2-\beta)} .
\end{aligned}
$$

Hence, if $0 \leq t_{1}<t_{2} \leq 1$, we have

$$
\begin{aligned}
& \left\|\operatorname{Tu}\left(t_{2}\right)-T u\left(t_{1}\right)\right\| \\
& \leq\left|\frac{L}{\Gamma(\alpha)}\left(\frac{t_{2}^{\alpha}-t_{1}^{\alpha}}{\alpha}+\frac{\left(t_{2}^{2}-t_{1}^{2}\right)\left(1-\lambda \eta^{\alpha-1}\right)}{2-2 \lambda \eta}\right)\right|+\left|\frac{L\left(2-\lambda \eta+\lambda \eta^{\alpha-1}\right)}{(1-\lambda \eta) \Gamma(2-\beta) \Gamma(\alpha)}\left(t_{2}^{1-\beta}-t_{1}^{1-\beta}\right)\right| \\
& \quad \leq \frac{L}{\Gamma(\alpha)}\left(\left|\frac{t_{2}^{\alpha}-t_{1}^{\alpha}}{\alpha}+\frac{\left(t_{2}^{2}-t_{1}^{2}\right)\left(1-\lambda \eta^{\alpha-1}\right)}{2-2 \lambda \eta}\right|+\left|\frac{2-\lambda \eta+\lambda \eta^{\alpha-1}}{(1-\lambda \eta) \Gamma(2-\beta)}\left(t_{2}^{1-\beta}-t_{1}^{1-\beta}\right)\right|\right) .
\end{aligned}
$$

If $-r \leq t_{1}<t_{2} \leq 0$, we have

$$
\left\|T u\left(t_{2}\right)-T u\left(t_{1}\right)\right\|=\left\|\varphi\left(t_{2}\right)-\varphi\left(t_{1}\right)\right\| .
$$

If $-r \leq t_{1}<0<t_{2} \leq 1$, then

$$
\left\|T u\left(t_{2}\right)-T u\left(t_{1}\right)\right\| \leq \frac{1}{\Gamma(\alpha)}\left|\frac{t_{2}^{\alpha}}{\alpha}+\frac{t_{2}^{2}\left(1-\lambda \eta^{\alpha-1}\right)}{2-2 \lambda \eta}\right|+\left\|\varphi\left(t_{1}\right)\right\|+\frac{L t_{2}^{1-\beta}}{\Gamma(2-\beta)} .
$$

In any case, it implies that $\left\|T u\left(t_{2}\right)-T u\left(t_{1}\right)\right\| \rightarrow 0$ as $t_{2} \rightarrow t_{1}$, i.e., for any $\epsilon>0$, there exists $\delta>0$, independent of $t_{1}, t_{2}$ and $u$, such that $\left|T u\left(t_{2}\right)-T u\left(t_{1}\right)\right| \leq \epsilon$, whenever $\left|t_{2}-t_{1}\right|<\delta$. Therefore $T: X \rightarrow X$ is completely continuous. The proof is completed.

For convenience, we denote

$$
\begin{aligned}
& M=\frac{1}{\Gamma(\alpha)}\left(\frac{1}{\alpha}+\frac{1}{2-2 \lambda \eta}+\frac{\lambda \eta^{\alpha-2}}{2-2 \lambda \eta}\right), \\
& N=\frac{1}{\Gamma(2-\beta) \Gamma(\alpha)}\left(1+\frac{1+\lambda \eta^{\alpha-1}}{1-\lambda \eta}\right) .
\end{aligned}
$$

\section{Theorem 3.2 Assume that}

$\left(\mathrm{H}_{3}\right)$ There exists a constant $p>0$ such that $|f(t, \mu, v)-f(t, \bar{\mu}, \bar{v})| \leq p(|\mu-\bar{\mu}|+|v-\bar{v}|)$ for each $\mu, \bar{\mu} \in C_{r}, v, \bar{v} \in \mathbb{R}$. If

$$
p<(M+N)^{-1}
$$

then boundary value problem (1.1) and (1.2) has a unique solution.

Proof Consider the operator $T: X \rightarrow X$ defined by (3.2). Clearly, the fixed point of the operator $T$ is the solution of boundary value problem (1.1) and (1.2). We will use the Banach contraction principle to prove that $T$ has a fixed point. We first show that $T$ is a 
contraction. For each $t \in[0,1]$,

$$
\begin{aligned}
|T u(t)-T \bar{u}(t)| \leq & \int_{0}^{1}|G(t, s)| f\left(s, u_{s},{ }^{C} D^{\beta} u(s)\right)-f\left(s, \bar{u}_{s},{ }^{C} D^{\beta} \bar{u}(s)\right) \mid d s \\
\leq \leq & \frac{p\|u-\bar{u}\|}{\Gamma(\alpha)}\left(\int_{0}^{t}(t-s)^{\alpha-1} d s+\frac{(\alpha-1) t^{2}}{2-2 \lambda \eta} \int_{0}^{1}(1-s)^{\alpha-2} d s\right. \\
& \left.+\frac{\lambda(\alpha-1) t^{2}}{2-2 \lambda \eta} \int_{0}^{\eta}(\eta-s)^{\alpha-2} d s\right) \\
\leq & \frac{p\|u-\bar{u}\|}{\Gamma(\alpha)}\left(\frac{t^{\alpha}}{\alpha}+\frac{t^{2}}{2-2 \lambda \eta}+\frac{t^{2} \lambda \eta^{\alpha-2}}{2-2 \lambda \eta}\right) \\
\leq & \frac{p\|u-\bar{u}\|}{\Gamma(\alpha)}\left(\frac{1}{\alpha}+\frac{1}{2-2 \lambda \eta}+\frac{\lambda \eta^{\alpha-2}}{2-2 \lambda \eta}\right) \\
\leq & p\|u-\bar{u}\| M .
\end{aligned}
$$

By a similar method, we get

$$
\begin{aligned}
\left|{ }^{C} D^{\beta} T u(t)-{ }^{C} D^{\beta} T \bar{u}(t)\right|= & \left|\frac{1}{\Gamma(1-\beta)} \int_{0}^{t}(t-s)^{-\beta}\left(T u^{\prime}(s)-T \bar{u}^{\prime}(s)\right) d s\right| \\
\leq & \frac{1}{\Gamma(1-\beta)} \int_{0}^{t}(t-s)^{-\beta}\left(\int_{0}^{1}\left|\frac{\partial}{\partial s} G(s, z)\right| f\left(z, u_{z},{ }^{C} D^{\beta} u(z)\right)\right. \\
& \left.-f\left(z, \bar{u}_{z},{ }^{C} D^{\beta} \bar{u}(z)\right) \mid d z\right) d s \\
\leq & \frac{p\|u-\bar{u}\|}{\Gamma(1-\beta)} \int_{0}^{t}(t-s)^{-\beta}\left(\int_{0}^{1}\left|\frac{\partial}{\partial s} G(s, z)\right| d z\right) d s .
\end{aligned}
$$

In view of the definition of $G(t, s)$, we obtain

$$
\begin{aligned}
& \int_{0}^{1}\left|\frac{\partial G(t, s)}{\partial t}\right| d s \\
& \quad \leq \int_{0}^{t}\left|\frac{(t-s)^{\alpha-2}}{\Gamma(\alpha-1)}\right| d s+\frac{2(\alpha-1) t}{2-2 \lambda \eta} \int_{0}^{1}\left|\frac{(1-s)^{\alpha-2}}{\Gamma(\alpha)}\right| d s+\frac{2 \lambda(\alpha-1) t}{2-2 \lambda \eta} \int_{0}^{\eta}\left|\frac{(\eta-s)^{\alpha-2}}{\Gamma(\alpha)}\right| d s \\
& \quad \leq \frac{t^{\alpha-1}}{\Gamma(\alpha)}+\frac{t}{(1-\lambda \eta) \Gamma(\alpha)}+\frac{t \lambda \eta^{\alpha-1}}{(1-\lambda \eta) \Gamma(\alpha)} \\
& \quad \leq \frac{1}{\Gamma(\alpha)}\left(1+\frac{1+\lambda \eta^{\alpha-1}}{1-\lambda \eta}\right) .
\end{aligned}
$$

Hence,

$$
\begin{aligned}
\left|{ }^{C} D^{\beta} T u(t)-{ }^{C} D^{\beta} T \bar{u}(t)\right| & \leq \frac{p\|u-\bar{u}\|}{\Gamma(1-\beta)} \int_{0}^{t} \frac{(t-s)^{-\beta}}{\Gamma(\alpha)}\left(1+\frac{1+\lambda \eta^{\alpha-1}}{1-\lambda \eta}\right) d s \\
& \leq \frac{p\|u-\bar{u}\|}{\Gamma(1-\beta)} \frac{1}{\Gamma(\alpha)} \frac{1}{1-\beta}\left(1+\frac{1+\lambda \eta^{\alpha-1}}{1-\lambda \eta}\right) \\
& \leq \frac{p\|u-\bar{u}\|}{\Gamma(2-\beta) \Gamma(\alpha)}\left(1+\frac{1+\lambda \eta^{\alpha-1}}{1-\lambda \eta}\right) \\
& \leq p\|u-\bar{u}\| N .
\end{aligned}
$$


Clearly, for each $t \in[-r, 0]$, we have $|T u(t)-T \bar{u}(t)|=0$. Therefore, by (3.7) and (3.9), we get

$$
\|T u-T \bar{u}\| \leq p\|u-\bar{u}\| M+p\|u-\bar{u}\| N \leq p\|u-\bar{u}\|(N+M) \leq\|u-\bar{u}\|
$$

and $T$ is a contraction. As a consequence of the Banach contraction principle, we get that $T$ has a fixed point which is a solution of boundary value problem (1.1) and (1.2).

\section{Example}

In this section, we will present some examples to illustrate our main results.

Example 4.1 Consider boundary value problems of the following fractional functional differential equations:

$$
\begin{aligned}
& { }^{C} D^{\alpha} u(t)=\frac{e^{t}-1}{36}+\frac{e^{-t}}{110}\left|u_{t}\right|^{k_{1}}+\frac{e^{-t}}{143}\left|{ }^{C} D^{\beta} u(t)\right|^{k_{2}}, \\
& u(0)=u^{\prime}(0)=0, \quad u^{\prime}(1)=\frac{6}{5} u^{\prime}\left(\frac{1}{3}\right),
\end{aligned}
$$

where ${ }^{C} D^{\alpha},{ }^{C} D^{\beta}$ denote Caputo fractional derivatives, $2<\alpha<3,0<\beta<1, t \in(0,1)$.

Choose $\lambda=\frac{6}{5}, \eta=\frac{1}{3}, \phi(t)=\frac{e^{t}-1}{36}, a=\frac{e^{-t}}{110}, b=\frac{e^{-t}}{143}$ and

$$
f\left(t, u_{t},{ }^{C} D^{\beta} u(t)\right)=\frac{e^{t}-1}{36}+\frac{e^{-t}}{110}\left|u_{t}\right|^{k_{1}}+\frac{e^{-t}}{143}\left|{ }^{C} D^{\beta} u(t)\right|^{k_{2}} .
$$

Then, for $t \in(0,1)$, we have

$$
\left|f\left(t, u_{t},{ }^{C} D^{\beta} u(t)\right)\right| \leq \phi(t)+a\left|u_{t}\right|^{k_{1}}+b\left|{ }^{C} D^{\beta} u(t)\right|^{k_{2}} .
$$

For $0<k_{1}, k_{2}<1,\left(\mathrm{H}_{1}\right)$ is satisfied and for $k_{1}, k_{2}>1,\left(\mathrm{H}_{2}\right)$ is satisfied. Therefore, by Theorem 3.1, boundary value problem (4.1) and (4.2) has a solution.

Example 4.2 Consider boundary value problems of the following fractional functional differential equations:

$$
\begin{aligned}
& { }^{C} D^{\alpha} u(t)=\frac{\left|u_{t}\right|+\left|{ }^{C} D^{\beta} u(t)\right|}{\left(6+9 e^{t}\right)\left(1+\left|u_{t}\right|+\left|{ }^{C} D^{\beta} u(t)\right|\right)}, \\
& u(0)=u^{\prime}(0)=0, \quad u^{\prime}(1)=\frac{7}{6} u^{\prime}\left(\frac{3}{8}\right),
\end{aligned}
$$

where ${ }^{C} D^{\alpha},{ }^{C} D^{\beta}$ denote Caputo fractional derivatives, $2<\alpha<3,0<\beta<1, t \in(0,1)$.

Choose $\lambda=\frac{7}{6}, \eta=\frac{3}{8}$ and

$$
f\left(t, u_{t},{ }^{C} D^{\beta} u(t)\right)=\frac{\left|u_{t}\right|+\left|{ }^{C} D^{\beta} u(t)\right|}{\left(6+9 e^{t}\right)\left(1+\left|u_{t}\right|+\left|{ }^{C} D^{\beta} u(t)\right|\right)} .
$$

Set

$$
f(t, \mu, \nu)=\frac{|\mu|+|v|}{\left(6+9 e^{t}\right)(1+|\mu|+|v|)} .
$$


Let $\mu, \bar{\mu} \in C_{r}, v, \bar{v} \in \mathbb{R}$. Then for each $t \in[0,1]$,

$$
\begin{aligned}
|f(t, \mu, v)-f(t, \bar{\mu}, \bar{\nu})| & =\frac{1}{6+9 e^{t}}\left|\frac{|\mu|+|\nu|}{1+|\mu|+|\nu|}-\frac{|\bar{\mu}|+|\bar{\nu}|}{1+|\bar{\mu}|+|\bar{\nu}|}\right| \\
& =\frac{|\mu+\bar{\mu}|-|\nu-\bar{\nu}|}{\left(6+9 e^{t}\right)(1+|\bar{\mu}|+|\bar{\nu}|)(1+|\mu|+|\nu|)} \\
& \leq \frac{1}{6+9 e^{t}}(|\mu+\bar{\mu}|-|\nu-\bar{\nu}|) \\
& \leq \frac{1}{15}(|\mu+\bar{\mu}|-|\nu-\bar{\nu}|) .
\end{aligned}
$$

For each $t \in[-1,0]$,

$$
\begin{aligned}
|f(t, \mu, \nu)-f(t, \bar{\mu}, \bar{\nu})| & =\frac{1}{6+9 e^{t}}\left|\frac{|\mu|+|\nu|}{1+|\mu|+|\nu|}-\frac{|\bar{\mu}|+|\bar{\nu}|}{1+|\bar{\mu}|+|\bar{\nu}|}\right| \\
& =\frac{|\mu+\bar{\mu}|-|\nu-\bar{\nu}|}{\left(6+9 e^{t}\right)(1+|\bar{\mu}|+|\bar{v}|)(1+|\mu|+|\nu|)} \\
& \leq \frac{1}{6+9 e^{t}}(|\mu+\bar{\mu}|-|\nu-\bar{\nu}|) \\
& \leq \frac{1}{6}(|\mu+\bar{\mu}|-|\nu-\bar{v}|) .
\end{aligned}
$$

Thus the condition $\left(\mathrm{H}_{3}\right)$ holds with $p=\frac{1}{15}$. For $\lambda=\frac{7}{6}, \eta=\frac{3}{8}$, we have

$$
\begin{aligned}
M & =\frac{1}{\Gamma(\alpha)}\left(\frac{1}{\alpha}+\frac{1}{2-2 \times \frac{7}{6} \times \frac{3}{8}}+\frac{\frac{7}{6}\left(\frac{3}{8}\right)^{\alpha-2}}{2-2 \times \frac{7}{6} \times \frac{3}{8}}\right) \\
& =\frac{1}{\Gamma(\alpha)}\left(\frac{1}{\alpha}+\frac{8}{9}+\frac{28}{27}\left(\frac{3}{8}\right)^{\alpha-2}\right), \\
N & =\frac{1}{\Gamma(2-\beta) \Gamma(\alpha)}\left(1+\frac{1+\frac{7}{6}\left(\frac{3}{8}\right)^{\alpha-1}}{1-\frac{7}{6} \times \frac{3}{8}}\right) \\
& =\frac{1}{\Gamma(2-\beta) \Gamma(\alpha)}\left(1+\frac{1+\frac{7}{6}\left(\frac{3}{8}\right)^{\alpha-1}}{\frac{9}{16}}\right) .
\end{aligned}
$$

By $2<\alpha<3,0<\beta<1$, we have

$$
\frac{1}{3} \leq \frac{1}{\alpha}<\frac{1}{2}
$$

and

$$
M>\frac{1}{\Gamma(\alpha)} \frac{131}{54}, \quad N>\frac{32}{9 \Gamma(2-\beta) \Gamma(\alpha)} .
$$

It implies that

$$
p=\frac{1}{15}<0.167<(M+N)^{-1} .
$$

Then by Theorem 3.2, boundary value problem (4.3) and (4.4) has a unique solution. 


\section{Competing interests}

The authors declare that they have no competing interests.

\section{Authors' contributions}

The authors declare that the study was realized in collaboration with the same responsibility. All authors read and approved the final manuscript.

\section{Acknowledgements}

The authors sincerely thank the reviewers for their valuable suggestions and useful comments that have led to the present improved version of the original manuscript. This research is supported by the Natural Science Foundation of China $(11071143,60904024,61174217)$, Natural Science Outstanding Youth Foundation of Shandong Province (JQ201119) and supported by Shandong Provincial Natural Science Foundation (ZR2012AM009, ZR2010AL002, ZR2011AL007), also supported by Natural Science Foundation of Educational Department of Shandong Province (J11LA01)

\section{Received: 6 December 2012 Accepted: 7 February 2013 Published: 22 February 2013}

\section{References}

1. Podlubny, I: Fractional Differential Equations. Academic Press, San Diego (1999)

2. Kilbas, A, Srivastava, $H$, Trujillo, J: Theory and Applications of Fractional Differential Equations. Elsevier, Amsterdam (2006)

3. Oldham, K, Spanier, J: The Fractional Calculus. Academic Press, New York (1974)

4. Miller, K, Ross, B: An Introduction to the Fractional Calculus and Fractional Differential Equation. Wiley, New York (1993)

5. Samko, S, Kilbas, A, Marichev, O: Fractional Integral and Derivative, Theory and Applications. Gordon and Breach, Switzerland (1993)

6. Chang, Y-K, Zhang, R, N'Guérékata, GM: Weighted pseudo almost automorphic mild solutions to fractional differential equations. Comput. Math. Appl. 64(10), 3160-3170 (2012)

7. Agarwal, RP, Benchohra, M, Hamani, A: Boundary value problems for fractional differential equations. Georgian Math. J. 16, 401-411 (2009)

8. Zhao, Y, Sun, S, Han, Z, Li, Q: The existence of multiple positive solutions for boundary value problems of nonlinear fractional differential equations. Commun. Nonlinear Sci. Numer. Simul. 16(4), 2086-2097 (2011)

9. Zhao, Y, Sun, S, Han, Z, Li, Q: Positive solutions to boundary value problems of nonlinear fractional differential equations. Abstr. Appl. Anal. 2011, 1-16 (2011)

10. Zhao, Y, Sun, S, Han, Z, Zhang, M: Positive solutions for boundary value problems of nonlinear fractional differential equations. Appl. Math. Comput. 217, 6950-6958 (2011)

11. Feng, W, Sun, S, Han, Z, Zhao, Y: Existence of solutions for a singular system of nonlinear fractional differential equations. Comput. Math. Appl. 62(3), 1370-1378 (2011)

12. Rehman, $M$, Khan, $R$, Asif, $N$ : Three point boundary value problems for nonlinear fractional differential equations. Acta Math. Sci. 31B(4), 1337-1346 (2011)

13. Diethelm, K: The Analysis of Fractional Differential Equations. Springer, Berlin (2010)

14. Sun, S, Zhao, Y, Han, Z, Li, Y: The existence of solutions for boundary value problem of fractional hybrid differential equations. Commun. Nonlinear Sci. Numer. Simul. 17, 4961-4967 (2012)

15. Sun, $\mathrm{S}, \mathrm{Zhao}, \mathrm{Y}, \mathrm{Han}, \mathrm{Z}, \mathrm{Xu}, \mathrm{M}$ : Uniqueness of positive solutions for boundary value problems of singular fractional differential equations. Inverse Probl. Sci. Eng. 20, 299-309 (2012)

16. Benchohra, M, Henderson, J, Ntouyas, S, Ouahab, A: Existence results for fractional order functional differential equations with infinite delay. J. Math. Anal. Appl. 338, 1340-1350 (2008)

17. Lakshmikantham, V, Vatsala, A: Basic theory of fractional differential equations. Nonlinear Anal. 69, 2677-2682 (2008)

18. Lakshmikantham, $\vee$, Vatsala, A: Theory of fractional differential inequalities and applications. Commun. Appl. Anal. 11 395-402 (2007)

19. Lakshmikantham, V: Theory of fractional functional differential equations. Nonlinear Anal. 69, 3337-3343 (2008)

20. Lakshmikantham, V, Devi, J: Theory of fractional differential equations in Banach space. Eur. J. Pure Appl. Math. 1, 38-45 (2008)

21. Agarwal, RP, Zhou, Y, He, Y: Existence of fractional neutral functional differential equations. Comput. Math. Appl. 59, 1095-1100 (2010)

22. Agarwal, RP, Zhou, Y, Wang, J, Xian, N: Fractional neutral functional differential equations with causal operators in Banach spaces. Math. Comput. Model. 54, 1440-1452 (2011)

23. Sun, $\mathrm{S}, \mathrm{Li}, \mathrm{Q}, \mathrm{Li}, \mathrm{Y}$ : Existence and uniqueness of solutions for a coupled system of multi-term nonlinear fractional differential equations. Comput. Math. Appl. 64, 3310-3320 (2012)

24. Li, Q, Sun, S, Zhao, P, Han, Z: Existence and uniqueness of solutions for initial value problem of nonlinear fractional differential equations. Abstr. Appl. Anal. 2012, 1-14 (2012)

25. Maraaba, T, Baleanu, D, Jarad, F: Existence and uniqueness theorem for a class of delay differential equations with left and right Caputo fractional derivatives. J. Math. Phys. 49, 483-507 (2008)

26. Zhou, Y, Jiao, F, Li, J: Existence and uniqueness for $p$-type fractional neutral differential equations. Nonlinear Anal. TMA 71(7-8), 2724-2733 (2009)

27. Zhou, Y, Jiao, F, Li, J: Existence and uniqueness for fractional neutral differential equations with infinite delay. Nonlinear Anal. TMA 71(7-8), 3249-3256 (2009)

28. Wang, J, Zhou, Y, Wei, W: A class of fractional delay nonlinear integrodifferential controlled systems in Banach spaces. Commun. Nonlinear Sci. Numer. Simul. 16, 4049-4059 (2011)

29. Zhou, Y, Tian, Y, He, Y: Floquet boundary value problems of fractional functional differential equations. Electron. J. Qual. Theory Differ. Equ. 50, 1-13 (2010)

30. Bai, C: Existence of positive solutions for a functional fractional boundary value problem. Abstr. Appl. Anal. 2010, 1-13 (2010) 
31. Ouyang, Z, Chen, YM, Zou, SL: Existence of positive solutions to a boundary value problem for a delayed nonlinear fractional differential system. Bound. Value Probl. 2011, 1-17 (2011)

32. Bai, C: Existence of positive solutions for boundary value problems of fractional functional differential equations. Electron. J. Qual. Theory Differ. Equ. 30, 1-14 (2010)

33. Ahmad, B, Alsaedi, A: Nonlinear fractional differential equations with nonlocal fractional integro-differential boundary conditions. Bound. Value Probl. 2012, 1-10 (2012)

34. Zhao, Y, Chen, H, Huang, L: Existence of positive solutions for nonlinear fractional functional differential equation. Comput. Math. Appl. 64, 3456-3467 (2012)

35. Su, X: Positive solutions to singular boundary value problems for fractional functional differential equations with changing sign nonlinearity. Comput. Math. Appl. 64, 3425-3435 (2012)

36. Guo, D, Lakshmikantham, V: Nonlinear Problems in Abstract Cone. Academic Press, Orlando (1988)

doi:10.1186/1687-2770-2013-38

Cite this article as: Li et al.: Three-point boundary value problems of fractional functional differential equations with delay. Boundary Value Problems 2013 2013:38.

\section{Submit your manuscript to a SpringerOpen ${ }^{\circ}$ journal and benefit from:}

- Convenient online submission

Rigorous peer review

- Immediate publication on acceptance

- Open access: articles freely available online

- High visibility within the field

- Retaining the copyright to your article 